\title{
Prevalence of Mycobacterium tuberculosis Strains Isolated from Both Pulmonary and Extra Pulmonary Samples and Their Resistance to Rifampicin: A Study from Kolkata and Surrounding Suburbs
}

\author{
Soma Chakraborty ${ }^{1}$, Arindam Chakraborty ${ }^{1}$, Tathagata Talukder1, Mita Mukherjee1, \\ Tridip Chatterjee ${ }^{2^{*}}$ \\ ${ }^{1}$ Department of Microbiology, Suraksha Diagnostics DD-18/1, Kolkata, India \\ ${ }^{2}$ Suraksha Genomics (R \& D Division of Suraksha Diagnostics) DD-18/1, Kolkata, India \\ Email: ctridip@gmail.com, tridip.academic@gmail.com
}

Received 9 April 2016; accepted 3 June 2016; published 6 June 2016

Copyright (C) 2016 by authors and Scientific Research Publishing Inc.

This work is licensed under the Creative Commons Attribution International License (CC BY). http://creativecommons.org/licenses/by/4.0/

(c) (i) Open Access

\section{Abstract}

Tuberculosis (TB) is one of the major causes of morbidity and mortality worldwide. In India, nearly 1.8 million new cases of TB are reported annually, which accounts for a fifth of new cases in the world-greater than in any other country. Anti-tubercular drugs (ATDs) have been used for decades, and widespread resistance to them is a very serious public health concern in any part of the world. Aim of this study was to determine the prevalence of Rifampicin (the first line Anti-TB drug) resistance among both pulmonary and extra-pulmonary samples tested positive for Mycobacterium tuberculosis and thereby predict the prevalence of Multi-drug resistant (MDR) tuberculosis in Kolkata and its Suburban regions. All 331 randomly collected clinical samples (both Pulmonary and Extra Pulmonary) were initially screened by Zeihl-Neelsen AFB staining followed by culture on BacT/Alert 3D system and on Lowenstein-Jensen medium and the positive samples were subjected to detection of Mycobacterium tuberculosis complex (MTBC) and simultaneous analysis of Rifampicin resistance by Xpert MTB/RIF assay. Out of the $51(15.40 \%)$ culture positive samples, $13.7 \%$ of pulmonary samples and $9.09 \%$ of extra-pulmonary samples were Rifampicin resistant. The prevalence of Rifampicin resistant TB in our study is high and the possible reasons can be mixing of new as well as retreatment cases and smaller sample size but, yet it can help Government and public health regulatory bodies to formulate adequate strategies to fight against drug resistant tuberculosis, especially in this part of the world.

\footnotetext{
"Corresponding author.

How to cite this paper: Chakraborty, S., Chakraborty, A., Talukder, T., Mukherjee, M. and Chatterjee, T. (2016) Prevalence of Mycobacterium tuberculosis Strains Isolated from Both Pulmonary and Extra Pulmonary Samples and Their Resistance to Rifampicin: A Study from Kolkata and Surrounding Suburbs. Journal of Tuberculosis Research, 4, 61-71.

http://dx.doi.org/10.4236/jtr.2016.42008
} 


\section{Keywords}

\section{Tuberculosis (TB), Multi Drug Resistant TB (MDR-TB), Rifampicin Resistant TB, GeneXpert}

\section{Introduction}

Tuberculosis (TB) is one of the major causes of morbidity and mortality worldwide. In India, about 1.8 million new cases of TB are reported annually, which accounts for a fifth of new cases in the world-a greater number than in any other country [1].

Anti-tuberculosis (TB) drug resistance is a major public health problem that threatens progress made in TB care and control worldwide. Drug resistance arises due to improper and irrational use of anti-tubercular drugs (ATDs) in chemotherapy of drug-susceptible TB patients. This improper use is a result of a number of actions including administration of improper treatment regimens and failure to ensure that patients complete the whole course of treatment. Essentially, drug resistance indicates a weakness in TB control program in that area. A patient who develops active disease with a drug-resistant TB strain can transmit this form of TB to other individuals. Strategies used for the clinical management of patients infected with drug-resistant Mycobacterium tuberculosis complex (MTBC) are different, therefore, prompt detection, isolation, and implementation of alternate anti-tubercular treatment regimens are necessary for suitable management [2] [3]. Moreover, early detection of such cases is of utmost importance in preventing spread of resistant bugs in the community.

In a study by Surandra K. Sharma et al. a total of 177 cases of sputum positive pulmonary tuberculosis were enrolled between 2008 and 2009 of which only two cases of MDR-TB were detected. Thus, the prevalence of MDR-TB among newly diagnosed pulmonary tuberculosis patients was 1.1 percent [4].

Anti-TB medicines have been used for decades, and resistance to them is widespread. TB strains may be resistant to one or more ATDs. Rifampicin-resistant tuberculosis (RR-TB) is caused by tubercle bacilli that displays in vitro resistance to Rifampicin, one of the most effective ATDs, requiring longer treatment regimens, institution of alternative (the 2nd line) medications, higher incidences of adverse effects and decreased compliance than patients with Rifampicin-susceptible disease. Multidrug-resistant tuberculosis (MDR-TB) is a form of Rifampicin-resistant TB that has additional resistance to isoniazid, the first-line bactericidal ATD and extensively drug-resistant TB (XDR-TB) is a form of multi-drug resistant tuberculosis that responds to even fewer available medicines, including the two most effective classes of the "second-line" anti-TB medicines.

Globally, 5\% of TB cases were estimated to have had multidrug-resistance (MDR-TB) in 2014. Drug resistance surveillance data showed that an estimated 480,000 people developed MDR-TB in 2014 and 190,000 people died as a result of MDR-TB. Extensively drug-resistant TB (XDR-TB) has been reported by 105 countries in 2014. On average, an estimated 9.7\% of people with MDR-TB have XDR-TB [5].

Rifampicin resistance is regarded as an alternative method for detection of MDR-TB as a large proportion of Rifampicin-resistant strains also display resistance to isoniazid (INH). Although other genetic methods such as LPA (Line Probe Assay) can detect both RIF and INH resistance, it can at times underestimate the INH resistance by missing many genetic resistance locations. And despite the fact that culture identification and drug sensitivity testing (DST) are gold standard methods of detection of drug resistance, one cannot ignore the fact that they take very long duration of time. Hence, Rifampicin resistance can be used to satisfactorily indicate MDR-TB in populations with high burden [6].

\section{Study Design}

This study was initiated to determine the prevalence of Mycobacterium tuberculosis and its resistance to Rifampicin among patients in Kolkata and its surrounding regions from May 2015 to January 2016 as a research project under Suraksha Genomics, a division of Suraksha Diagnostics. The samples were processed as per Figure 1.

All available samples were first decontaminated and concentrated by Petroff's method [7] for initial screening by ZN stain and subsequent culture. All samples irrespective of stain positive or negative were subjected to culture in both egg based solid media as well as in liquid culture media. Molecular detection of Mycobacterium species and resistance to Rifampicin were then studied by Gene Xpert from culture positive colony isolates. Ge- 


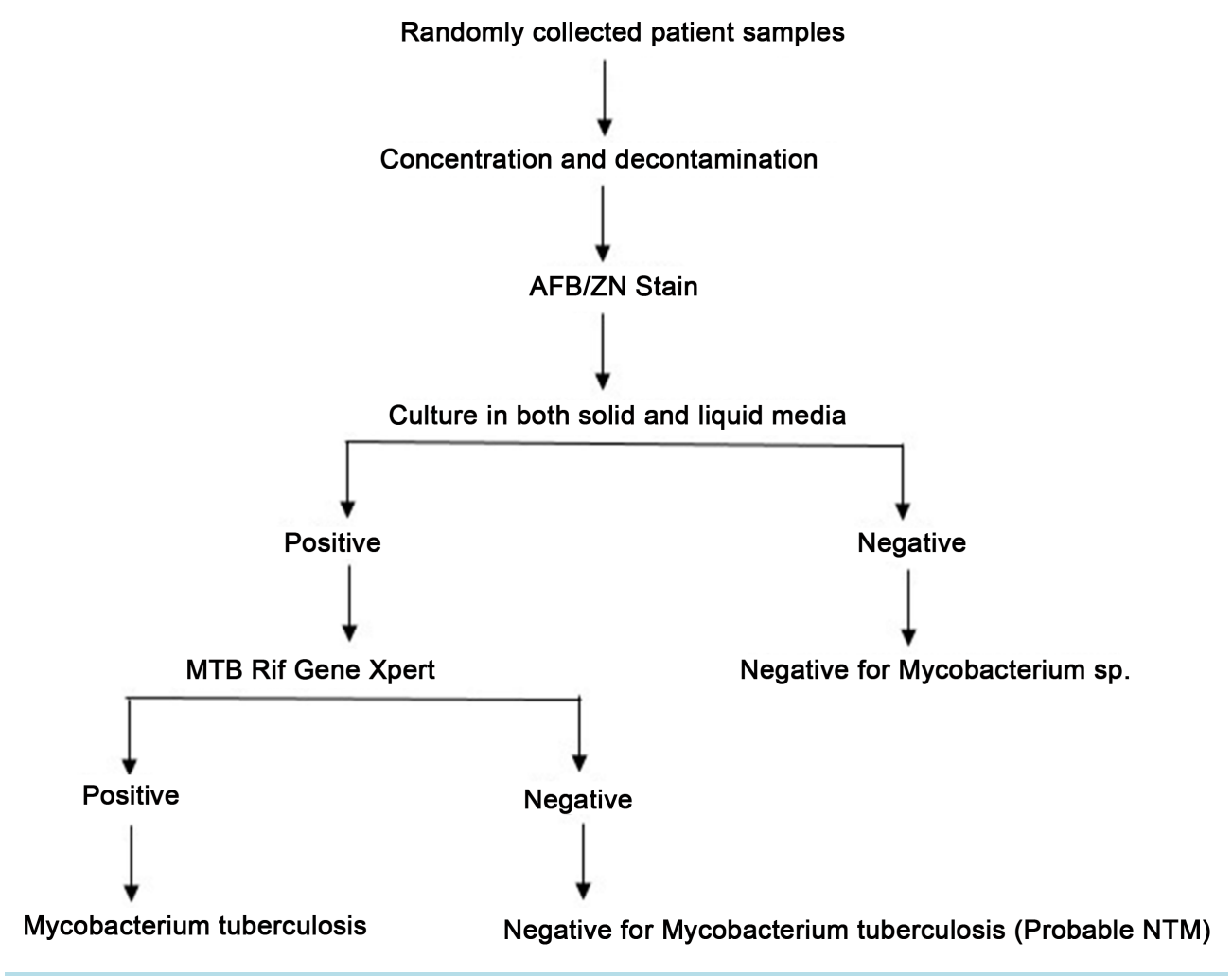

Figure 1. Sample processing flow-chart.

neXpert positive cases were considered as Mycobacterium tuberculosis complex (MTBC) and negative cases were as Non-Tubercular Mycobacterium sp (NTM).

\section{Patient Selection Strategy}

A total of 331 properly collected samples (91 pulmonary and 240 extra pulmonary) from patients with clini$\mathrm{cal} /$ radiological suspicion of Tubercular infection were included in this study. We included both pulmonary (like deeply expectorated freshly collected sputum samples, free of saliva, blood and food contamination and bronchial alveolar lavage samples) and extra-pulmonary samples (such as all body fluids, tissue, urine, pus, aspirates etc.). Samples were included irrespective of the treatment status of the patients (e.g. both new suspected cases as well as post-treatment cases). Patients were finally included on the basis of availability of consent forms. Any patient without consent was excluded from the study. For this study, the patient population included patients from all strata of society irrespective of economic status and means of livelihood. All samples showing evidence of contamination with saliva (determined by Bartlett's grading system) [8] were excluded from our study. The growths of Mycobacterium species other than M. tuberculosis (MOTT/NTM) were also excluded. We excluded the whole blood samples as well as swab samples for TB diagnosis in this study as per standard guidelines.

Inclusion criteria: Both pulmonary (like deeply expectorated freshly collected sputum samples, free of saliva, blood and food contamination and bronchial alveolar lavage samples) and extra-pulmonary samples (such as all body fluids, tissue, urine, pus, aspirates etc.) were included. All samples were selected on the basis of availability of consent.

Exclusion criteria: Swabs, Blood, grossly contaminated samples with saliva and blood were excluded from our study.

\section{Methods}

All the samples were subjected to initial screening for the presence of Acid Fast Bacilli by Zeihl-Neelsen staining method followed by culture. All samples were decontaminated and concentrated by the modified Petroff's method [7]. Culture was done on liquid media (Biomerieux TB BacT/Alert 3D) as well as on egg based solid 
media (Lowenstein-Jensen medium) and incubated aerobically at optimum temperature. As culture is considered to be the gold standard [9], the culture negative isolates were interpreted as Negative for Mycobacterium sp.

\section{Culture}

All clinical samples for TB/AFB culture requires a special pre-analytic treatment step, called decontamination and homogenization to let the tubercle bacilli free from mucus, cells or tissue and also to prevent overgrowth of any commensal organism that may be present in the specimen. This step was carried out by Sodium Hydroxide $(\mathrm{NaOH})$ methodby Modified Petroff's procedure for culture on solid media like LJ media and NALC-NaOH method for liquid culture in Bact/Alert. This provides a liquification-decontamination and specimen buffering procedure that maintains the viability and pathogenicity of Mycobacterium spp. NALC acts as a mucolytic agent whereas $\mathrm{NaOH}$ decontaminates other micro-organisms and final wash with phosphate buffer ensures that specimen is at optimum $\mathrm{pH}$ for culturing. Specimen pre-treatment and decontamination increases relative Acid fast Bacilli concentration and ensures its more sensitive detection during culture.

Each sample was mixed with equal volume of mucolytic reagents followed by centrifugation at 4000-5000 rpm. The pellets are collected and mixed with $1 \mathrm{ml}$ of distilled water. One loopfull (22 SWG Nichrome loops) this mixture was inoculated on each LJ medium slops (two slops per specimen). The slants were placed vertically for 1 week and horizontally for the next 5 weeks. ZN staining is done from any suspicious colonies grown on the LJ slants on a weekly basis till the end of routine duration of incubation of 6 weeks.

The automated liquid AFB culture vial contain middlebrook $7 \mathrm{H} 9$ broth, pancreatic digest of casein, bovine serum albumin, catalase and purified water, and are prepared with an atmospheric $\mathrm{CO}_{2}, \mathrm{~N}_{2}$ and $\mathrm{O}_{2}$ under vacuum. Each bottle was inoculated with $0.5 \mathrm{~mL}$ pellet-suspension after initial treatment with NALC-NaOH. Any bacteria if present in the vial will produce $\mathrm{CO}_{2}$ as respiratory metabolite. When growth of microorganism produces $\mathrm{CO}_{2}$ the colour of the gas permeable sensor installed at the bottom of the vial will turn lighter green or yellow which in turn will be detected by the optical sensor of the machine. All bottles signalled positive were subsequently confirmed by ZN staining for AFB.

Identification of Mycobacterium tuberculosis complex (MTBC) and Rifampicin susceptibility:

Hemi-nested real time PCR was used for detection of Mycobacterium tuberculosis complex (MTBC) and the Rifampicin resistance in the isolated strains. The GeneXpert MTB/RIF assay is a novel integrated diagnostic device that performs sample processing and hemi-nested real-time Polymerase Chain Reaction (PCR) analysis in a single hands-free step for the diagnosis of MTBC and rapid detection of Rifampicin (RIF) resistance in clinical specimens as well as in culture isolates [10] [11]. The Xpert MTB/RIF detects DNA sequences specific for $M y$ cobacterium tuberculosis and Rifampicin resistance by polymerase chain reaction [10] [12]. It is based on the Cepheid GeneXpert system which is a platform for rapid and simple-to-use nucleic acid amplification tests (NAAT).The Xpert ${ }^{\circledR}$ MTB/RIF purifies and concentrates Mycobacterium tuberculosis bacilli from sputum samples and then isolates genomic material from the captured bacteria by sonication and subsequently amplifies the genomic DNA by PCR.

Rifampicin resistance was detected by PCR amplification of the 81-bp fragment of the M. tuberculosis RNA polymerasebeta $(r p o B)$ gene and subsequent probing of this region for mutations that are associated with RIF resistance. The process identifies all the clinically relevant Rifampicin resistance inducing mutations in the RNA polymerase beta (rpoB) gene in the Mycobacterium tuberculosis genome in a real time format using fluorescent probes called molecular beacons.Sensitivity of GeneXpert is $97.2 \%$ and specificity is $98.3 \%$ (according to the manufacturer). Acceptability of GeneXpert is high due its rapid detection, a very little and simple pre-operational sample processing and almost no chance of contamination [10].

\section{Results}

331 clinical samples (91 pulmonary and 240 extra-pulmonary) were analyzed during the period of our study (Figure 2). Number of Positive (MTB detected) and Negative (MTB not detected) samples, screened through Ziehl-Neelsen AFB Staining procedure and culture by liquid media as well as by solid media are given in Table 1. The results of molecular detection of $r p o B$ gene (which confers rifampicin resistance), done by GeneXpert are summarized in Table 2. Figure 3 shows a 3+ positive (as per RNTCP grading) AFB stain (Z. N. stain). Figure 4 shows a GeneXpert report where MTB (Mycobacterium tuberculosis) is detected, while RIF (Rifampicin) re- 


\section{Distribution of Pulmonary and extra pulmonary samples}

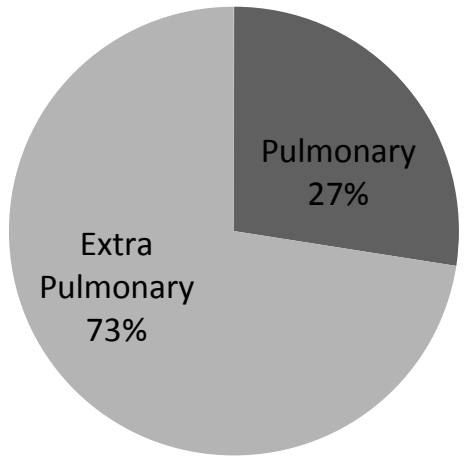

Figure 2. Distribution of Pulmonary and extra-pulmonary samples $(\mathrm{n}=331)$.

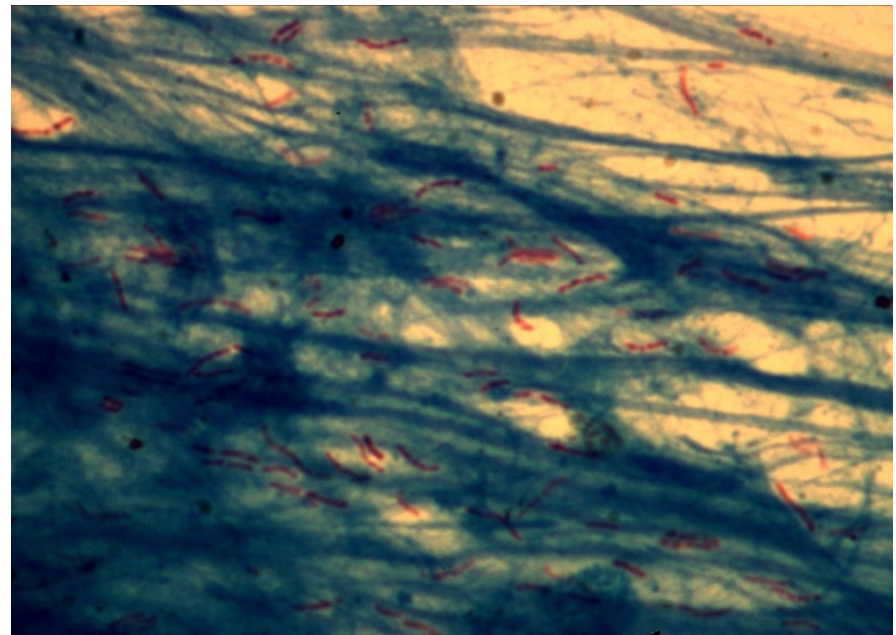

Figure 3. AFB positive smear (Under 100× oil magnification).

Table 1. Frequency of AFB grown in both liquid and solid media culture methods.

\begin{tabular}{cccc}
\hline Sample Type & Total Samples & AFB Culture Positive & AFB Culture negative \\
\hline Pulmonary & 91 & $30(32.9 \%)$ & $61(67.1 \%)$ \\
Extra Pulmonary & 240 & $22(9.2 \%)$ & $218(90.8 \%)$ \\
\hline
\end{tabular}

Table 2. Frequency of Rifampicin resistant cases in pulmonary and extra pulmonary samples.

\begin{tabular}{cccc}
\hline Sample Type & Total Samples & MTB Detected in XpertMTB/Rif & Rif Resistance Detected \\
\hline Pulmonary & 30 & 29 & $4(13.7 \%)$ \\
Extra Pulmonary & 22 & 22 & $2(9.09 \%)$ \\
\hline
\end{tabular}

sistance is not detected. Figure 5 shows a GeneXpert report where both MTB \& RIF resistance has been detected.

The results show that, out of 91 pulmonary samples, 28 (30.7\%) were positive for AFB by ZN staining and 30 samples $(32.9 \%)$ were both stain and culture positive. Out of these 30 culture positive isolates 29 samples (96.66\%) were GeneXpert positive for Mycobacterium tuberculosis Complex (MTBC) and 1 sample was culture and AFB positive but GeneXpert Negative for Mycobacterium tuberculosis Complex (MTBC). This was considered as Non-Tubercular Mycobacterium sp. (Speciation not done) (Figure 6). 
Whereas out of the 240 extra-pulmonary samples 13 (5.41\%) were positive for AFB by ZN staining and 23 samples (9.2\%) were stain and culture positive for Acid fast bacilli as well as detected as positive for Mycobacterium tuberculosis on GeneXpert (Table 3).

4 pulmonary samples (13.7\%) out of 29 positive GeneXpert cases were found to be Rifampicin resistant and 2 (8.69\%) out of $23 \mathrm{GeneXpert} \mathrm{positive} \mathrm{extra-pulmonary} \mathrm{samples} \mathrm{were} \mathrm{found} \mathrm{to} \mathrm{be} \mathrm{Rifampicin} \mathrm{resistant.}$

Sex wise distribution study showed $21(70 \%)$ out of the 29 MTBC positive pulmonary cases were males and 8 $(30 \%)$ were females. Whereas 9 cases $(39 \%)$ out of 23 MTB positive extra pulmonary cases were found males and 14 cases $(61 \%)$ were found females (Table 4, Figures 7-10).

$3(75 \%)$ out of 4 Rifampicin resistant pulmonary TBcases were found in male and $1(25 \%)$ was female, whereas both the MTB positive Rifampicin resistant extra-pulmonary cases were females (100\%) (Table 5).

We checked for the HIV status of all cases positive for tuberculosis of our study and found them to be negative for HIV by immunochromatographic assay (ICT).

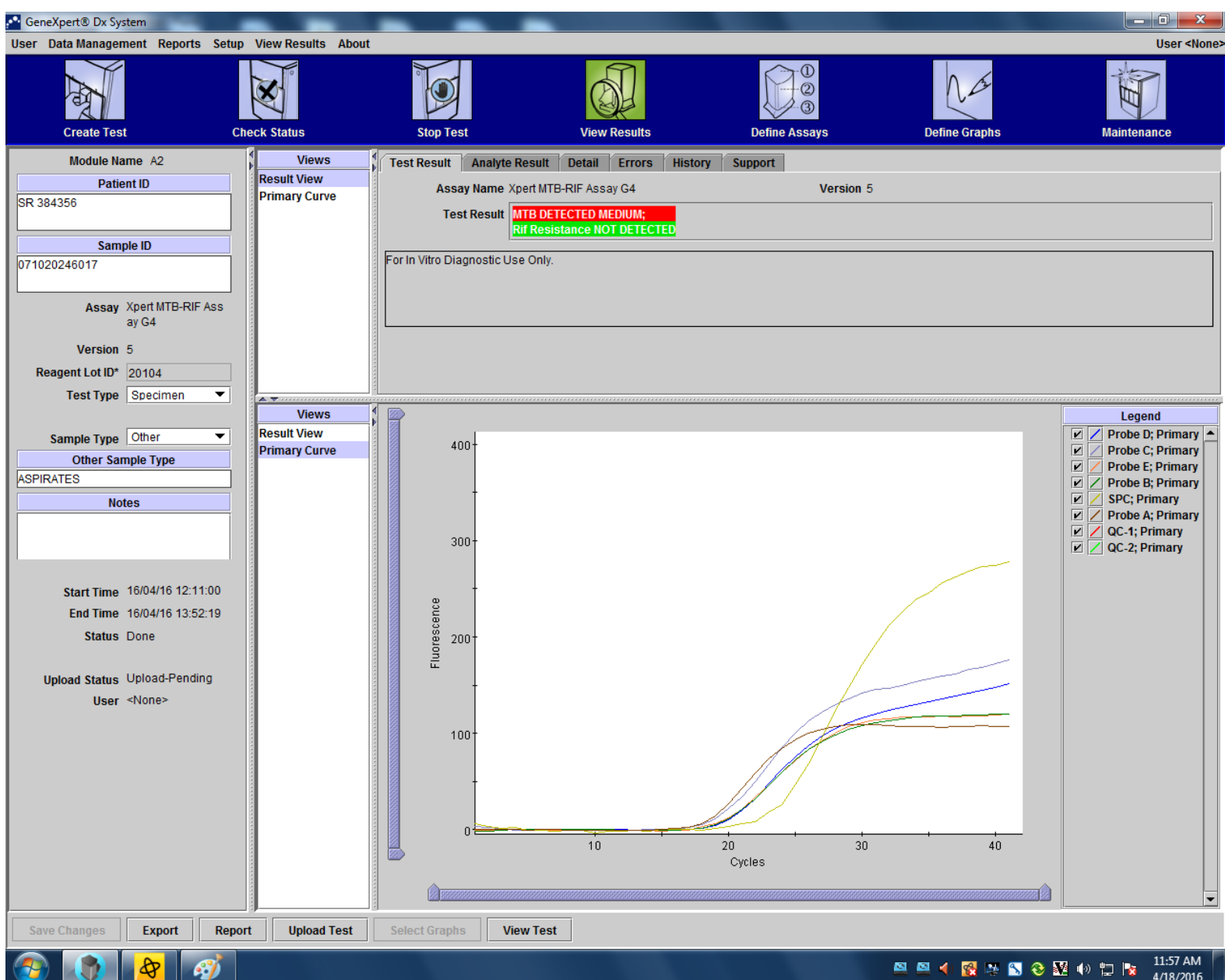

Figure 4. GeneXpert view: MTB detected, RIF resistance not detected.

Table 3. Correlation between AFB Stain and culture.

\begin{tabular}{rccccccc}
\hline & \multicolumn{3}{c}{ Pulmonary $(\mathrm{n}=91)$} & \multicolumn{3}{c}{ Extra Pulmonary $(\mathrm{n}=\mathbf{2 4 0})$} \\
\cline { 2 - 7 } & Stain $+\mathrm{ve}$ & Stain $-\mathrm{ve}$ & Total & Stain $+\mathrm{ve}$ & Stain $-\mathrm{ve}$ & Total \\
\hline Culture positive & 28 & 2 & 30 & 13 & 10 & 23 \\
Culture negative & 0 & 61 & 61 & 0 & 217 & 217 \\
\hline
\end{tabular}




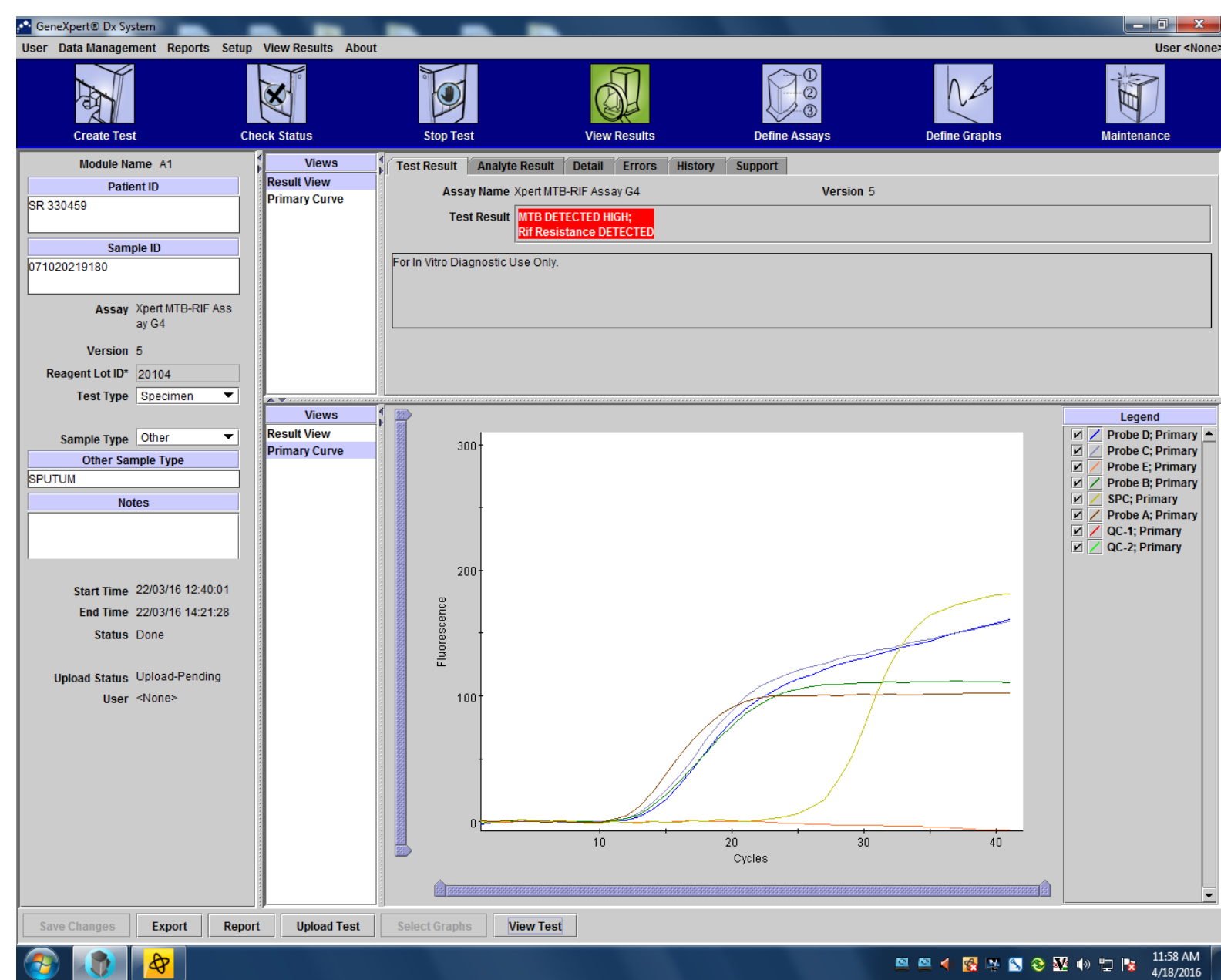

Figure 5. GeneXpert view: MTB detected, RIF resistance detected.

Table 4. Age and sex distribution of MTB positive cases.

\begin{tabular}{ccccc}
\hline \multirow{2}{*}{ Age distribution } & \multicolumn{2}{c}{ Pulmonary } & \multicolumn{2}{c}{ Extra Pulmonary } \\
\cline { 2 - 5 } & Male $(\mathbf{n}=\mathbf{2 1})$ & Female $(\mathbf{n}=\mathbf{8})$ & Male (n=9) & Female $(\mathbf{n}=\mathbf{1 4})$ \\
\hline$<20$ & $1(4.76 \%)$ & $1(11 \%)$ & $1(11 \%)$ & $3(21.42 \%)$ \\
$20-40$ & $8(38.09 \%)$ & $4(44 \%)$ & $8(89 \%)$ & $9(64.28 \%)$ \\
$40-60$ & $8(38.09 \%)$ & $2(23 \%)$ & 0 & $2(14.28 \%)$ \\
$>60$ & $4(19.04 \%)$ & $1(11 \%)$ & 0 & 0 \\
\hline
\end{tabular}

Table 5. Age and Sex distribution of MTB positive Rifampicin resistant cases.

\begin{tabular}{|c|c|c|c|c|}
\hline \multirow{2}{*}{ Age distribution } & \multicolumn{2}{|c|}{ Pulmonary } & \multicolumn{2}{|c|}{ Extra Pulmonary } \\
\hline & Male $(n=3)$ & Female $(n=1)$ & Male $(n=0)$ & Female $(n=2)$ \\
\hline$<20$ & $1(25 \%)$ & $1(100 \%)$ & 0 & 0 \\
\hline $20-40$ & $2(75 \%)$ & 0 & 0 & $2(100 \%)$ \\
\hline $40-60$ & 0 & 0 & 0 & 0 \\
\hline$>60$ & 0 & 0 & 0 & 0 \\
\hline
\end{tabular}




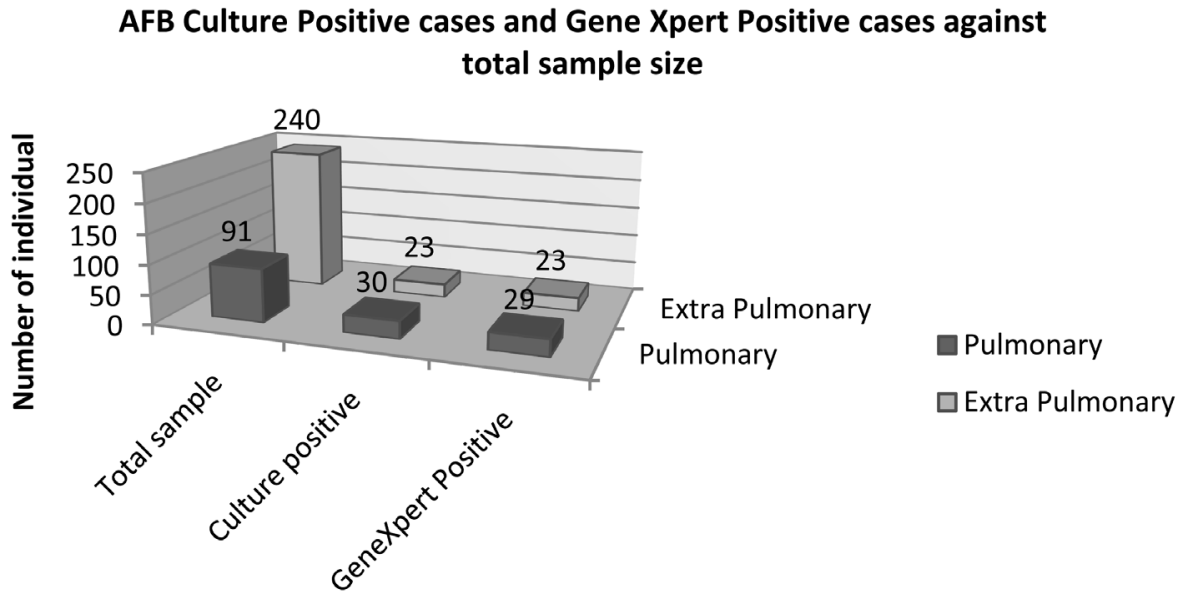

Figure 6. AFB culture positive cases and GeneXpert positive cases against total sample size.

Graphical Representation of Age \& sex wise distribution of patients suffering from Pulmonary Tuberculosis

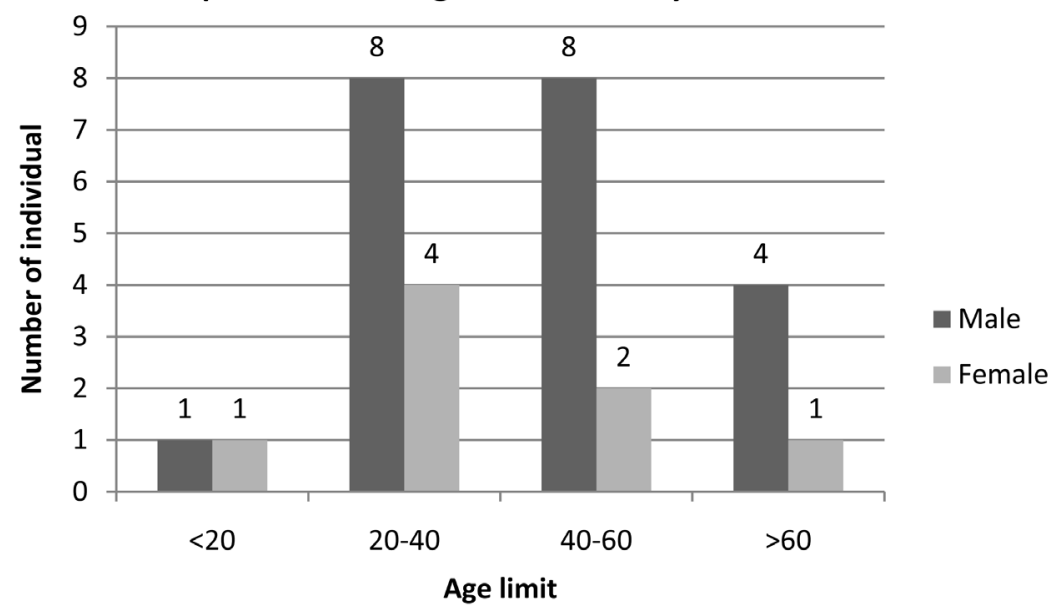

Figure 7. Graphical representation of age \& sex wise distribution of patients suffering from pulmonary tuberculosis $(n=29)$.

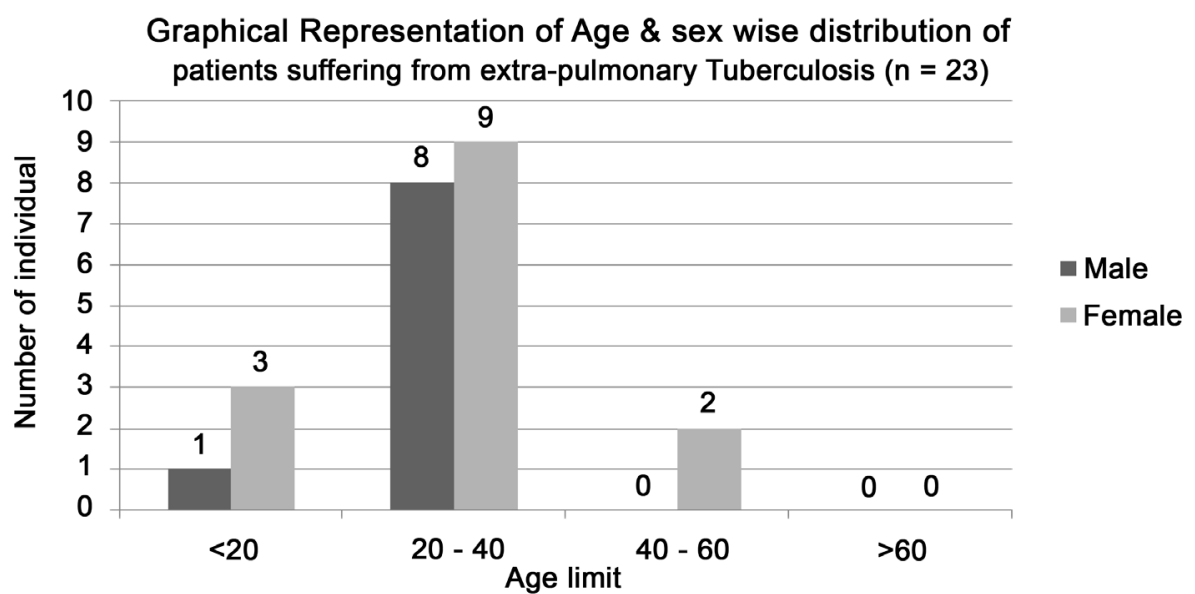

Figure 8. Graphical representation of age \& sex wise distribution of patients suffering from extrapulmonary tuberculosis $(\mathrm{n}=23)$. 


\section{Sex wise distributionm of MTB positive pulmonary samples}

$(n=29)$

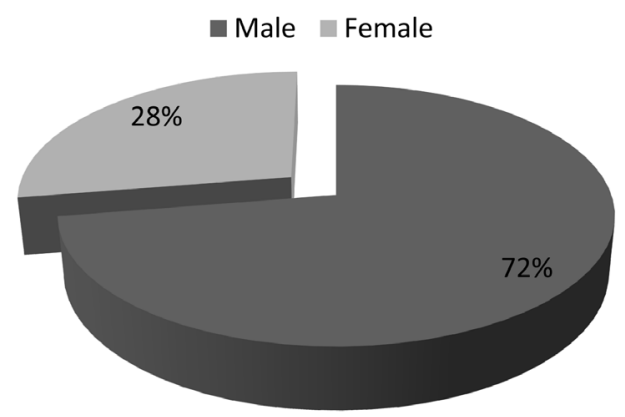

Figure 9. Sex wise distribution of MTB positive pulmonary samples.

\section{Sex wise distribution of MTB positive extra pulmonary samples $(n=23)$}

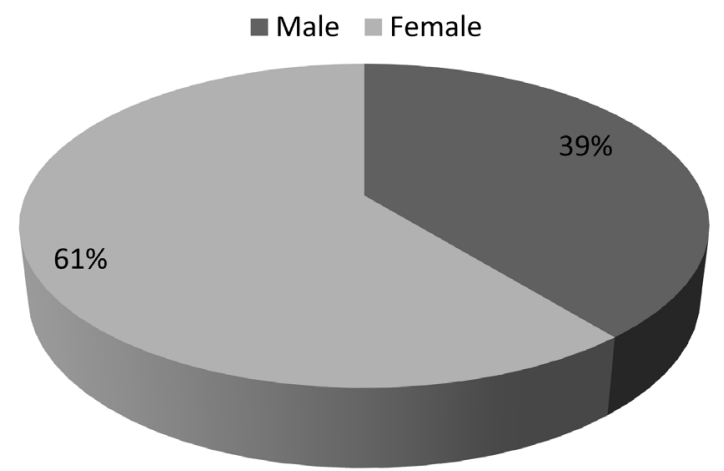

Figure 10. Sex wise distribution of MTB positive extra pulmonary samples.

\section{Discussion}

The reported prevalence of drug-resistant TB was found variable in different studies from around the world and in our country. In our study, out of the 331 clinical samples (both pulmonary and extra pulmonary), 51(15.4\%) were positive for Mycobacterium tuberculosis and the prevalence of Rifampicin resistance to TB was 13.7\% from all pulmonary samples and $8.69 \%$ from all extra-pulmonary samples. Different studies from around India have reported a Rifampicin mono-resistance at around 9\% [13] [14]. A study in 2011-2012 by Surajit Lahirietalreported a Rifampicin mono-resistance of 4.69\% [15] where as another study by M. Giridhar Kumar et al. shows 0\% Rifampicin resistance among MTB positive cases in 2010-2012 mainly from South India [16].

Many studies published from different parts of India have reported high MDR-TB prevalence, but mostly among first-time re-treatment patients with relapse, treatment after default, and treatment after failure [17] [18]. The possible reasons of a higher prevalence of drug resistance in our study can be, mixing of new as well as retreatment cases and smaller sample size.

Although many Indian studies have reported lower prevalence of Rifampicin mono-resistance from various parts of the country, in our study the higher rate can be due to a possible co-existence of INH resistance and the rate may be acting as a proxy to the local MDR-TB prevalence.

In our study majority of the pulmonary MTB infected male patients were within the age group of $20-60$ years and female patients, within the age group of 20 - 40 years. In the case of extra pulmonary samples too both the males and females were from the age group of $20-40$ years (Table 4).

And in case of Rifampicin resistance, majority of pulmonary cases found in males and females were also from the age group of 20 - 40 years. However we cannot conclude from here as because of the low sample size the values may be statistically insignificant. 
We tried to establish a relationship between drug resistant TB and HIV co-infection [19]. But in our study all TB positive cases (irrespective of their MDR-TB status) were HIV negative.

The emergence of resistance to drugs used to treat tuberculosis (TB), and particularly multidrug-resistant TB (MDR-TB), has become a significant public health problem in a number of countries and is an obstacle to effective TB control. In India, the available information from the several drug resistance surveillance studies conducted in the past suggest that the rate of MDR-TB is relatively low in India. However, this translates into a large absolute number of drug resistant TB cases and as yet the management of patients with MDR-TB is inadequate. Specific measures are being taken within the Revised National Tuberculosis Control Program (RNTCP) to address the MDR-TB problem through appropriate management of patients and strategies to prevent the propagation and dissemination of MDR-TB. Drug-resistant TB has microbial, clinical, and programmatic causes. From a microbiological perspective, the resistance is caused by a genetic mutation that makes a drug ineffective against the mutant bacilli. An inadequate or poorly administered treatment regimen allows drugresistant mutants to become the dominant strain in a patient infected with TB. However it should be stressed that MDR-TB is a man-made phenomenon-poor treatment, poor drugs and poor adherence lead to the development of MDR-TB.

This study will help us to screen the prevalence of MDR TB among the patients of Eastern India. India had an estimated 63,000 cases of notified multi-drug resistant tuberculosis (MDR-TB) in 2010, the highest in the South East Asia region, World Health Organization (WHO) has said. The MDR-TB prevalence is estimated to be 2.3 per cent among new cases and $12-17$ per cent among re-treatment cases. However, due to the size of the population and the number of TB cases reported annually, India ranks second among the 27 MDR-TB high-burden countries worldwide after China. According to WHO in 2014, 220,000 people died from TB in India, which is the highest in the world. The same report says that $2.1 \%$ cases in this emerging percentage are due to MDR-TB. Thus early detection of MDR-TB cases and initiation of appropriate treatment based on drug resistance testing can lower the burden of this deadly disease.

Throughout the course of this study we felt the need for further studies involving bigger number of samples, looking into the prevalence of Non-tubercular Mycobacteria (NTM) and testing resistance pattern with all five first-line drugs in future.

\section{Conclusion}

Tuberculosis has been a significant health hazard in India, but the change in its nature with higher prevalence of multi-drug resistance has made it all the more important for all of us to do more research in the this field and produce regional data. As a study of prevalence of drug resistance in TB from Kolkata, we believe that this study can help in the control of TB at the national level and probably can help us in the mapping drug resistant TB cases in this part of the country. This would in turn help in the planning of measures to tackle and control TB cases in such a manner so as to curb or rein in drug resistant TB (MDR-TB) cases.

\section{References}

[1] RNTCP (2010) New Delhi: Central TB Division. Directorate General of Health Services, Ministry of Health and Family Welfare, Status TB Report, Accessed on 7 February 2011. http://tbcindia.nic.in/view.php?lid=2919\&type=1

[2] Hasegawa, N., Miura, T., Ishii, K., Yamaguchi, K., Lindner, T.H., Merritt, S., et al. (2002) New Simple and Rapid Test for Culture Confirmation of Mycobacterium Tuberculosis Complex: A Multicenter Study. Journal of Clinical Microbiology, 40, 908-912. http://dx.doi.org/10.1128/JCM.40.3.908-912.2002

[3] Park, M.Y., Kim, Y.J., Hwang, S.H., Kim, H.H., Lee, E.Y., Jeong, S.H., et al. (2009) Evaluation of an Immunochromatographic Assay Kit for Rapid Identification of Mycobacterium tuberculosis Complex in Clinical Isolates. Journal of Clinical Microbiology, 47, 481-484.

[4] Sharma, S.K., Kaushik, G., Jha, B., George, N., Arora, S.K., Gupta, D., Singh, U., Hanif, M. and Vashisht, R.P. (2011) Prevalence of Multidrug-Resistant Tuberculosis among Newly Diagnosed Cases of Sputum-Positive Pulmonary tuberculosis. Indian Journal of Medical Research, 133, 308-311.

[5] World Health Organization (2015) Global Tuberculosis Report 2014. Cited 27 May. http://apps.who.int/iris/bitstream/10665/137094/1/9789241564809_eng.pdf

[6] Coovadia, Y.M., Mahomed, S., Pillay, M., Werner, L. and Mlisana, K. (2013) Rifampicin Mono-Resistance in Mycobacterium tuberculosis in KwaZulu-Natal, South Africa: A Significant Phenomenon in a High Prevalence TB-HIV Re- 
gion. PLoS ONE, 8, e77712.

[7] Murray, P.R., Baron, E.J., Pfaller, M.A., Tenover, F.C. and Yolken, R.H. (1999) Manual of Clinical Microbiology. 7th Edition, Kluwer Academic Publishers, Springer Publication Group, 107-108. http://link.springer.com/article/10.1023\%2FA\%3A1007025717379

[8] Correlation of Sputum Gram's Stain and Culture in Lower Respiratory Tract Infections Anuradha Mokkapati 1, Madhavi Yalamanchili 2. IOSR Journal of Dental and Medical Sciences (IOSR-JDMS), 8, 6-9.

[9] Cruickshank, R., Duguid, I.P., Maimion, B.P. and Swain, R.H.A. (1975) Medical Microbiology. 12th Edition, Vol. 2, Churchill Livingstone, Edinburgh, 390.

[10] Helb, D., et al. (2010) Rapid Detection of Mycobacterium tuberculosis and Rifampin Resistance by Use of On-Demand, Near-Patient Technology. Journal of Clinical Microbiology, 48, 229-237. http://dx.doi.org/10.1128/JCM.01463-09

[11] Blakemore R., et al. (2010) Evaluation of the Analytical Performance of the Xpert MTB/RIF Assay. Journal of Clinical Microbiology, 48, 2495-2501. http://dx.doi.org/10.1128/JCM.00128-10

[12] Van Rie, A., Page-Shipp, L., Scott, L., Sanne, I. and Stevens, W. (2010) Xpert® MTB/RIF for Point-of-Care Diagnosis of TB in High-HIV Burden, Resource-Limited Countries: Hype or Hope? Expert Review of Molecular Diagnostics, 10, 937-946. http://dx.doi.org/10.1586/erm.10.67

[13] Sethi, S., Mewara, A., Dhatwalia, S.K., Singh, H., Yadav, R., Singh, K., et al. (2013) Prevalence of Multidrug Resistance in Mycobacterium tuberculosis Isolates from HIV Seropositive and Seronegative Patients with Pulmonary Tuberculosis in North India. BMC Infectious Diseases, 13, 137. http://dx.doi.org/10.1186/1471-2334-13-137

[14] Pradhan, N., Desai, S., Kagal, A., Dharmashale, S., Bharadwaj, R., Ghorpade, S., et al. (2013) Patterns of TB DrugResistance in a Tertiary Care Facility in Pune, India. Journal of Clinical Microbiology, 2, 123.

[15] Lahiri, S., Mukherjee, A., Hazra, S., Jana, P., Roy, S. and Saha, B.K. (2015) First-Line Anti-Tubercular Drug Resistance of Mycobacterial Strains from Re-Treatment Cases that Were Smear-Positive at 4th Month Onwards under the Revised National Tuberculosis Control Program. Lung India, 32, 127-131. http://dx.doi.org/10.4103/0970-2113.152619

[16] Kumar, M.G., Vishuvardhana, R.K. and Setty, C.R. (2014) Study of Prevalence of Multi-Drug Resistant Tuberculosis in a Tertiary Care Hospital. Journal of Applied Medical Sciences (SJAMS), 2, 269-273.

[17] Gupta, S., Bandyopadhyay, D., Gupta, S., Sadhukhan, S. and Banerjee, S. (2012) A Sociodemographic Study of Multidrug Resistant Tuberculosis Cases from DOTS Clinics of Kolkata. Journal of the Indian Medical Association, 110, 723-725.

[18] Almeida, D., Rodigues, C., Udwadia, Z.F., Lalvani, A., Gothi, G.D., Mehta, P., et al. (2003) Incidence of MultidrugResistant Tuberculosis in Urban and Rural India and Implications for Prevention. Clinical Infectious Diseases, 36, 152154. http://dx.doi.org/10.1086/374931

[19] Spellman, C.W., Matty, K.J. and Weis, S.E. (1998) A Survey of Drug-Resistant Mycobacterium tuberculosis and Its Relationship to HIV Infection. AIDS, 12, 191-195. http://dx.doi.org/10.1097/00002030-199802000-00009 